\title{
Repensando la sostenibilidad en la economía social y solidaria desde la perspectiva de género: trayectoria de una experiencia santafesina
}

\author{
Rethinking of Sustainability in the Social and Solidarity \\ Economy from a Gender Perspective: Trajectory of a Santa \\ Fe experience
}

Luisina María Logiodice', Maricel Lorena Massera $\bigotimes^{2}$

${ }^{1}$ Instituto de Humanidades y Ciencias Sociales del Litoral (unL-Conicet), Santa Fe, Argentina. 2Universidad Autónoma de Entre Ríos, Argentina.

maricel.massera@conicet.gov.ar

Recibido: 4 de abril del $2017 \quad$ Aprobado: 18 de julio del $2017 \quad$ Disponible en línea: 14 de enero del 2018

Cómo citar este artículo: Logiodice, L. M. y Massera, M. L. (2018). Repensando la sostenibilidad en la economía social y solidaria desde la perspectiva de género. Trayectoria de una experiencia santafesina. Cooperativismo \& Desarrollo, 25(112), 05-17. doi: https://doi.org/10.16925/co.v25i112.2037.

\section{Resumen}

Propósito: este artículo se propone analizar la trayectoria y reconfiguración de una experiencia asociativa de pequeños productores y de productoras agrícolas de una zona semirural de la provincia de Santa Fe (Argentina).

Descripción: se indaga acerca de la sostenibilidad —en sentido plural— de este tipo de prácticas de la economía social y solidaria (Esys), así como de la participación de las mujeres en estos espacios. Esto posibilita integrar la dimensión de género en un análisis que problematiza las posibilidades de permanencia de dichas prácticas laborales.

Punto de vista: se parte de las discusiones actuales en torno a las concepciones de la sostenibilidad en la esys como nuevas formas de trabajo asociativas, colectivas y familiares, al incorporar la perspectiva de género como una mirada necesaria en los procesos socioeconómicos para, finalmente, analizar a la luz de estas conceptualizaciones el caso bajo estudio.

Conclusión: en este tipo de organizaciones, y en términos de sostenibilidad, resulta indispensable considerar los lazos y vínculos de confianza que se generan al interior de estas, de modo que permitan sostener arreglos asociativos y la cohesión grupal, además del aporte y el vínculo que tiene el ámbito privado del hogar, en tanto ámbito en que confluyen los trabajos de producción y de reproducción que posibilita sostener los niveles de vida de los integrantes.

Palabras clave: economía social y solidaria, género, sostenibilidad plural, trabajo. 


\title{
Rethinking of Sustainability in the Social and Solidarity Economy from a Gender Perspective: Trajectory of a Santa Fe Experience
}

\begin{abstract}
Purpose: This article aims to analyze the trajectory and reconfiguration of an associative experience of small agricultural producers in a semi-rural area of the province of Santa Fe in Argentina.

Description: The article explores the sustainability —in a plural sense - of this type of Social and Solidarity Economy (SSE) practices, as well as the participation of women in these spaces. The above makes it possible to integrate the gender dimension into an analysis of the permanence possibilities of these laboral practices.

Point of view: The study's starting point lies in current discussions about conceptions of sustainability in the SSE as new forms of associative, collective and familiar work, incorporating the gender perspective as a necessary approximation in the socio-economic processes, to then analyze the case study in the light of these conceptualizations.

Conclusion: It is essential to consider the connections and bonds of trust generated within the SSE organizations, which allow to sustain associative pacts and group cohesion, in addition to the contribution and linkage given by the private sphere of home, which makes it possible to sustain its members living standards.
\end{abstract}

Keywords: social and solidarity economy, gender, plural sustainability, work.

\section{Repensando a sustentabilidade na economia social e solidária a partir da perspectiva de gênero: trajetória de uma experiência de Santa Fé (Argentina)}

\section{Resumo}

Propósito: este artigo pretende analisar a trajetória e a reconfiguração de uma experiência associativa de pequenos produtores e produtoras agrícolas de uma zona semirrural da província de Santa Fé (Argentina).

Descrição: questiona-se a sustentabilidade —em sentido plural— desse tipo de práticas da Economia Social e Solidária (ESeS), bem como a participação das mulheres nesses espaços. 0 anterior possibilita integrar a dimensão de gênero a uma análise sobre as possibilidades de permanência de tais práticas laborais.

Ponto de vista: parte-se das discussões atuais em torno dos conceitos de sustentabilidade na ESeS, como novas formas de trabalho associativo, coletivo e familiar, integrando a perspectiva de gênero como aproximação necessária nos processos socioeconômicos para, finalmente, à luz desses conceitos, analisar o caso de estudo.

Conclusão: resulta indispensável considerar os laços de confiança que são gerados no interior das organizações da ESeS, que permitem manter pactos associativos e coesão grupal, além da contribuiç̧ão e vínculo do âmbito privado do lar, que possibilita manter os níveis de vida dos integrantes.

Palavras-chave: economia social e solidária, gênero, sustentabilidade plural, trabalho. 


\section{Introducción ${ }^{1}$}

Durante las últimas décadas en América Latina, han comenzado a resurgir $-\mathrm{y}$ de forma crecientedistintas expresiones y procesos socioeconómicos alternativos al modo de producción capitalista, orientados por principios autogestivos y solidarios (Casalis, 2007). Existe una coincidencia notoria acerca del contexto y las causas por las que estas experiencias surgen, en el que se reconocen, principalmente, las transformaciones del capitalismo global desde la década de los setenta del siglo xx, la crisis de los Estados latinoamericanos y el desmantelamiento de estructuras de integración social, entre otros (Coraggio, 1998a y 1998b; Salvia, 2007). Esta es una situación que se profundiza en la década de los noventa ante el aumento de la situación de pobreza, exclusión social y pérdida de empleo que fuerza a generar un conjunto de expresiones y prácticas económicas, las cuales a su vez se han acompañado y promovido de forma creciente desde el ámbito de la política pública.

Al considerar la particularidad del contexto en el que emergen este tipo de prácticas socioeconómicas, así como la posibilidad que significan en términos de acceso al trabajo mercantil - aunque informal en muchos casos-, resulta pertinente interrogarse acerca de las posibilidades de permanencia que estas presentan. Diversos estudios han abordado dichas posibilidades a partir de la consideración de diferentes aspectos y niveles de análisis. Algunos han focalizado su abordaje en aquellos factores que promueven o limitan el funcionamiento de los emprendimientos de la Esys (Abramovich, 2008; Abramovich y Vázquez, 2004); otros, en cambio, han considerado la idea de sostenibilidad (Hintze, 2009; Hoop, 2012). Sin embargo, en general no se integra la perspectiva de género como enfoque que permita observar en qué medida la Esys es sostenible en términos de igualdad.

Toma relevancia, por lo tanto, considerar no solo cómo estas organizaciones posibilitan resolver

\footnotetext{
1 Una versión preliminar de este trabajo fue presentada en el viII Congreso Latinoamericano de Estudios del Trabajo, realizado del 3 al 5 agosto del 2016 en la Facultad de Ciencias Económicas de la Universidad de Buenos Aires. Asimismo, este análisis se enmarca en las tareas vinculadas al proyecto de extensión de interés social "Promoción del comercio justo como herramienta de fortalecimiento de organizaciones de pequeños productores y de otras organizaciones sociales en el Gran Santa Fe", financiado por la Universidad Nacional del Litoral.
}

las necesidades de reproducción, sino también atender a la (re)configuración de los vínculos sociales, familiares y de género que estos procesos generan. Así, resulta necesario preguntarnos por las relaciones de género en estos ámbitos de trabajo, en tanto permiten poner de manifiesto la relación producción-reproducción, al visibilizar el trabajo no remunerado necesario para el cuidado de la vida y reconocer las relaciones de poder que atraviesan la división de tareas y riquezas.

En este sentido, este trabajo se interesa por el análisis de la emergencia de nuevas formas de trabajo asociativas, colectivas y familiares que se dieron en Argentina en las últimas décadas, así como en dar cuenta de las potencialidades y las limitantes de estas experiencias en términos de sostenibilidad e igualdad. Específicamente, nos proponemos describir y analizar la trayectoria de la experiencia La Raíz de la provincia de Santa Fe, y su posterior reconfiguración en el grupo Familias Campesinas. ${ }^{2}$

La Raíz surge a partir de un grupo de pequeños productores y productoras de las localidades de Desvío Arijón y Caima de la provincia de Santa Fe, como canal de comercialización de los productos de sus huertas agroecológicas. Desde hace más de un año, sin embargo, comienzan a organizarse de manera autónoma un grupo de familias pertenecientes a este grupo a fin de constituir una nueva iniciativa de coordinación comercial: Familias Campesinas. Esta experiencia da cuenta de un recorrido que nos permite profundizar e interrogarnos acerca de la sostenibilidad de este tipo de prácticas de la economía social y solidaria, así como indagar sobre la participación de las mujeres en estos espacios.

La metodología que se utilizó es de tipo cualitativa y se realizaron entrevistas semiestructuradas dirigidas a miembros de esta organización.

\section{Debates en torno a la sostenibilidad en la economía social y solidaria}

Existe actualmente un debate en torno a la sostenibilidad de las experiencias asociativas autogestionadas de la Esys, ya que se trata de una noción que aún se encuentra en construcción, y se refiere a "la

2 Los nombres de las organizaciones y sus integrantes fueron cambiados, a fin de resguardar el anonimato de instituciones y sujetos. 
capacidad de perdurar en el tiempo de cierta actividad, proceso o institución" (Vázquez, 2010, p. 98).

En principio, se identifican dos posturas frente a la idea de sostenibilidad en este tipo de experiencias: quienes afirman que, para alcanzar esta condición, estas experiencias deben aumentar sus capacidades con el propósito de competir en los mercados; y quienes creen que dicha sostenibilidad depende del desarrollo de instituciones y políticas basadas en principios económicos distintos como son la reciprocidad, la retribución, la administración doméstica y la planificación (Vázquez, 2010).

Desde la primera perspectiva, se sostiene la necesidad de que dichas prácticas alcancen una capacidad competitiva en el mercado - y con ello la autogeneración de ingresos-, para lo cual es imprescindible su promoción por parte del Estado (Singer, 2007); mientras que, desde la segunda mirada, autores como Coraggio (2008) sostienen que no es posible evaluar un emprendimiento desde una lógica puramente microeconómica sin incorporar y evaluar el contexto socioeconómico, cultural y político. Esto implica abarcar sus multidimensiones a nivel meso, para lo cual es necesario incorporar el concepto de sostenibilidad socioeconómica:

Éste refiere a una economía social y solidaria en proceso de transición, y que admitela vía de aparentes "subsidios" económicos generalizados (educación, capacitación, exceptuación de impuestos, sistemas de salud, etc.) a partir del principio de redistribución progresiva por parte de la economía pública, así como aportes de trabajo u otros recursos (trabajo voluntario, redes de ayuda mutua, uso de la vivienda para la producción, etc.) muchas veces basados en relaciones de reciprocidad y no computados como costos. (Coraggio, 2008, p. 51)

Así, se espera que este tipo de experiencias puedan, por un lado, cubrir sus gastos a partir de los ingresos que obtienen del mercado, y por el otro, lograr la reproducción de sus miembros (Coraggio, 2008).

Dadas las características particulares de estas prácticas, se reconoce que contemplan una racionalidad específica - a diferencia de otro tipo de emprendimientos (Gaiger, 2007) - , y que se encuentran atravesadas, por tanto, por distintos principios de comportamiento.

Polanyi (2011) identifica tres principios que se dan en las relaciones económicas entre los hombres: el principio de intercambio (regido por la lógica mercantil y de la equivalencia entre productos); el principio de la redistribución (en el cual una autoridad centraliza los recursos y los redistribuye hacia el resto de la comunidad); y, finalmente, el principio de la reciprocidad (vinculado a la idea de que en las relaciones económicas no se vinculan agentes económicos, sino personas que están insertas en comunidades, en ciertas tramas sociales, con lazos sociales que preexisten). Las transferencias de bienes y servicios regidos por estas dos últimas lógicas no implican una retribución material, por lo que formarían una esfera no-monetaria de la economía (Dos Santos, Matos Filho, Medeiros de Araujo, Chaves-Meireles y da Silva, 2013, p. 98).

Desde esta perspectiva, se sostiene que estos principios siempre y naturalmente han existido en toda sociedad, los cuales a su vez trascienden y superan la reducción de la economía a simple economía de mercado, y de toda empresa económica a empresa capitalista (Dos Santos et al., 2013). La Esys, en cuanto fenómeno social situado en el tiempo, debería pensarse desde la perspectiva de una economía plural en la que a su vez conviven tres subsistemas: lo público, lo privado y lo social, y en la que existe una "pluralidad" de lógicas involucradas en sus relaciones (Ghibaudi, 2003, p. 2).

$\mathrm{Al}$ reconocer los emprendimientos de Esys por fuera de una lógica reduccionista de organización del trabajo, en tanto también intervienen y se articulan las lógicas de reciprocidad y domesticidad, resulta necesario pensar también su sostenibilidad en términos plurales (França-Filho y Laville, 2005; Dos Santos et al., 2013; Vázquez, 2010).

Siguiendo a Dos Santos et al. (2013), podemos destacar cinco dimensiones de la Esys: económica (Lisboa, 2005; Polanyi, 2011); política (França-Filho y Laville, 2005; Sen, 2000); ambiental (Kraychete, 2012, Gaiger, 2012, como se citan en Dos Santos et al., 2013); asociativa (Kraychete, 2008, como se cita en Dos Santos et al., 2013; Ray, 2000); y del capital social (Ray, 2000); perspectivas todas complementarias y no excluyentes.

La primera de ellas, la dimensión económica, hace referencia a la gestión de recursos financieros y no financieros utilizados por estas organizaciones, necesarios para convalidar la eficiencia de las actividades mercantiles, lo cual les permitirá reinvertir, mantenerse y expandirse (Lisboa, 2005, p. 109). Asimismo, se puede contemplar esta dimensión de manera más amplia al integrar el modo de producción y el régimen de empleo de la sociedad en que 
dichas unidades económicas se desarrollan (Hopp, 2012, p. 46). La dimensión política aparece como uno de los principales elementos de distinción de estas experiencias, al convertirse estas en espacios de expresión de la ciudadanía y fomento de la democracia, dada la horizontalidad de su gestión. Refiere no solo a la institucionalidad que adquieren las formas asociativas, sino también a las formas de participación sociopolítica que tienen estos actores con otros actores del sector (p. 47). Implica al mismo tiempo la existencia de políticas públicas dirigidas a su fortalecimiento y su fomento como actor relevante de desarrollo (Gaiger, 2007). La tercera dimensión hace referencia al ambiente y, con ello, a la existencia de las condiciones necesarias para que estos emprendimientos subsistan, tales como el acceso al espacio físico, a los medios de producción, al crédito, etc. (Dos Santos et al., 2013, p.101). La dimensión asociativa, como indicador de la sostenibilidad de estas iniciativas, implica dar cuenta de sus implicaciones en términos de compromisos y responsabilidades (Kraychete y Costa, 1999, como se citan en Dos Santos et al., 2013), al reconocer la autogestión como un principio clave de estas. Finalmente, la dimensión de capital social contempla la propensión de los individuos a asociarse, a generar lazos de confianza entre ellos e involucrarse en los asuntos de la comunidad (Hall, 1999, como se cita en Dos Santos et al., 2013), el cual actuaría como una fuerza de desarrollo endógeno (Ray, 2000). Una de las condiciones para sostener este atributo estaría dada por la existencia de empatía entre los integrantes, mecanismo por el cual es posible activar y sostener una cohesión social grupal, lo que se reflejaría en una mayor propensión a la sostenibilidad de estas experiencias (Dos Santos et al., 2013, pp. 103-104).

En definitiva, si se consideran las particularidades y lógicas implicadas en este tipo de prácticas, se reconocen las limitantes de un abordaje desde la racionalidad mercantil a la hora de lograr un real conocimiento de la trama que posibilita la sostenibilidad de estas organizaciones, por lo que resulta necesario avanzar hacia un análisis plural de dicha sostenibilidad.

\section{Aportes conceptuales para un análisis económico de género}

Los abordajes hasta aquí presentados en torno a la sostenibilidad proponen el reconocimiento de múltiples dimensiones. Sin embargo, estos no contemplan específicamente un enfoque de género que permita considerar de qué manera la división sexual del trabajo cristaliza situaciones de desigualdad e inequidad entre mujeres y hombres.

Carrasco (2016) plantea, en términos generales, que la Esys se sigue posicionando en el ámbito extradoméstico y por este motivo la necesidad de considerar la reproducción como proceso central. Para esto resulta pertinente el encuentro disciplinar entre la Esys y la economía feminista, destacado por diversas autoras (Matthaei, 2010; Quiroga, 2010). Reconocer el aporte que el ámbito no monetizado o no mercantil realiza a los procesos económicos, plantea la necesidad de integrar las relaciones de género al análisis sobre la sostenibilidad.

En ese sentido, resulta conveniente considerar la relación dinámica y de interdependencia entre el ámbito doméstico y mercantil, reconociendo que estos espacios que producen tanto bienes y servicios como cuidados, se estructuran bajo distintos tipos de relación, pero, principalmente, a través del trabajo (Carrasco, 2016, p. 36).

Según De Oliveira y Ariza (1997, p. 186), la segregación configura espacios diferenciales entre grupos sociales a partir de atributos particulares. Dicha distinción cristaliza esferas con competencias y autoridad que no son neutrales y fomentan un acceso desigual a recursos. En ese sentido, la división sexual del trabajo es aquella que distribuye de manera jerárquica e inequitativa los trabajos productivos y de reproducción, y se sostiene con base en aquellos atributos que culturalmente se construyen en torno a lo esperable de hombres y mujeres. Genera así posiciones diferenciales que redundan en menores grados de autonomía económica de las mujeres (Rodríguez-Enríquez, 2010), e institucionaliza una diferencia que sostiene un orden social de género.

Benería (1981) profundiza en algunas implicancias que son relevantes y vale la pena destacar. La división sexual del trabajo resulta de una asignación social por la que se han separado las esferas en las que se concentran, principalmente, hombres y mujeres. Sin embargo, la interrelación entre actividades reproductivas y productivas en el ámbito doméstico hace difícil su análisis a través de una separación clara entre ellas, ya que su vinculación varía según se trate de una familia urbana u otra que se ubica en la economía de subsistencia. Esos límites difusos permiten pensar 
que la división sexual del trabajo no solo condiciona la relación hogar-mercado, sino la forma en que esa relación se produce. En segundo lugar, la participación en el mercado de trabajo se ve condicionada por la división del trabajo, la cual a su vez reproduce y refuerza esta situación de subordinación en la esfera doméstica que conlleva en el caso de las mujeres la responsabilidad del trabajo de cuidado. En definitiva, la carga de trabajo que esto significa en términos de doble jornada dificulta además su propia organización del tiempo, en detrimento de sus condiciones de vida. Por último, se debe considerar una visión dinámica de esta división del trabajo, y destacar que las funciones productivas y reproductivas pueden variar de acuerdo con los cambios económicos y sociales. Esto entonces justifica la necesidad de analizar los modos de producción y sus efectos en la división del trabajo en ambos planos: el de la unidad doméstica y el de la actividad productiva.

Reconocer entonces el modo en que la división sexual del trabajo opera en la interrelación de la esfera productiva y la reproductiva-doméstica, implica adoptar una visión global y amplia que contemple como parte del circuito económico el trabajo de cuidado no remunerado. Picchio (2001) incorpora el proceso de reproducción social a los análisis tradicionales de la economía e integra así las relaciones de género en el sistema económico. La autora amplía el flujo circular de la renta ${ }^{3}$ al integrar el trabajo reproductivo no remunerado, a través de lo que denomina "espacio de desarrollo humano", ya que las acciones que allí se desarrollan se vinculan con el bienestar de las personas y no con la valorización de mercancías. Bajo esta propuesta detalla tres funciones del trabajo reproductivo:

- Ampliación de la renta monetaria y permitir extender los niveles de vida (transformación de bienes y servicios).

- Expansión de ese nivel de vida ampliado en forma de bienestar (disfrute de niveles específicos, convencionalmente adecuados, de educación, salud, vida social).

- Reducción de la población trabajadora a los efectivamente empleados (el trabajo reproductivo

3 Desde un enfoque macroeconómico, este diagrama, en su versión simple, grafica los flujos: real (factores, bienes y servicios) y monetario, que se dan en el mercado entre hogares y empresas. sirve de apoyo para la selección de las personas y las capacidades personales utilizadas en el proceso de producción).

Este análisis resulta interesante para el enfoque desde el cual se posiciona este trabajo al permitir, por un lado, considerar la división del trabajo al interior del hogar o la familia y, por otro, la funcionalidad económica del trabajo no remunerado en su vinculación con el sostenimiento de la producción de mercado. Permite reconocer el vínculo que permanece oculto entre trabajo de cuidado no remunerado con el sistema de producción capitalista, ya que es ese puente el que posibilita la transferencia de costos de dicha producción al interior del hogar, además que garantiza el proceso de reproducción social y lo deja libre a la órbita de lo privado (Carrasco, Borderías y Torns, 2011; Rodríguez-Enríquez, 2010; Picchio, 2001). Asimismo, este trabajo es absolutamente necesario para el mercado y la producción con relación al aspecto subjetivo y relacional del trabajo doméstico y de cuidado que permite la socialización de las personas que sostendrán el trabajo de mercado (Carrasco et al., 2011, p. 51).

\section{La sostenibilidad a partir de la trayectoria organizacional: la experiencia de La Raíz desde sus inicios}

La Raíz se inició a partir de un grupo de pequeños productores y productoras de zonas semirurales de la provincia de Santa Fe, quienes preocupados por los problemas derivados de los productos agrotóxicos utilizados en el cultivo en campos aledaños comenzaron a producir de manera agroecológica, con el objeto de autoabastecerse. A partir de esta situación, algunos de ellos establecieron contacto con otro grupo de huerteros - referente en este tipo de producción-, a fin de fomentar una nueva relación con el campo. De a poco surgió la idea de vender al público en la localidad y zonas cercanas e institucionalizar así una feria itinerante. De esta manera, el área sembrada comenzó a expandirse y los productos que vendían a diversificarse. Con idas y vueltas distintos productores se pusieron en contacto con La Raíz, tanto de forma activa como en un menor grado de participación en la organización. 
Como modo de organización, se decidió que cada feriante realizara un listado de los precios de todos sus productos (acordados con el resto de productores), que serían luego entregados al encargado de repartirlos. Este último adicionaba a cada uno de ellos un monto destinado a cubrir los gastos del traslado, del día de reparto, de teléfono y de Internet. En reuniones semanales, se debatían algunas de estas cuestiones (la cantidad de productos que cada uno llevaba, la calidad mínima requerida, de qué forma se hacía el traslado, en qué vehículos, etc.), aunque no todos los productores participaban de ellas:

No, no sabíamos de dónde eran. Pero sí entregaron sus producciones. Lo que reclamábamos era conocernos, hacer todo un círculo... de hecho habíamos programado hacer unas visitas mensuales y no, no tuvimos suerte. (Entrevistada 1, comunicación personal, 1 de julio de 2016)

\section{Asimismo, señala otra entrevistada:}

No, yo no tengo contacto con ellos, a las reuniones de la feria no voy porque no tengo tiempo y me queda lejos, imagínate si tengo que ir con la nena... tenemos un auto con mi marido, pero con el movimiento que tenemos me tendría que ir caminando... la llevo a la escuela a upa y vuelvo con la espalda dolorida ya. A la feria iba en un principio, pero ya después no. (Entrevistada 2, comunicación personal, 4 de julio 2015)

La Raíz aparecía como una alternativa para generar ingresos a familias que hasta ese momento solo podían ocuparse en huertas o campos privados. Los ingresos, sin embargo, no eran los mismos para todos los productores. Quienes contaban con mayores extensiones dedicadas a la producción fruti-hortícola (productos de mayor demanda), les fue posible con los ingresos de las ventas de las ferias satisfacer mayores necesidades. Sin embargo, este canal de comercialización no les impedía mantener ingresos de otras actividades, incluso de ventas que individualmente realizaran por fuera de la feria.

Nosotros con estas pequeñas producciones, si bien no te podés enriquecer, sí podés llevar algo de dinero a la casa para mejorar. Nosotros podemos consumir lo que producimos, pero necesitamos por ejemplo la ropa, el calzado, entonces nos sirve para vivir como campesinos. (Entrevistada 1, comunicación personal, 1 de julio de 2016)

Quienes realizaban otro tipo de producción (e. g. dulces y conservas, panificación, etc.) fueron quienes más dificultades tuvieron para obtener de la feria un ingreso suficiente que les permita sostener el hogar. Este fue un importante motivo por el que muchos de estos productores dejaron de participar de este espacio.

Por otra parte, se valoran articulaciones con distintas instituciones que de algún modo apoyaron las posibilidades de mejora del grupo y la organización. En particular, los vínculos con organismos del Estado ocurrían principalmente con el Instituto Nacional de Tecnología Agropecuaria (Inta), a través de la Secretaría de Agricultura Familiar. En su relación con este organismo se destacan la recepción de capacitaciones de diversos tipos (como de manipulación de alimentos, de envasado y presentación), así como el acompañamiento en la formalización de algunos de los integrantes a través del monotributo agropecuario, lo cual les permitió vender en comercios a los que antes no accedían. A través del vínculo con referentes territoriales de dicho organismo, a su vez, desde La Raíz, se consiguió un subsidio para la compra de algunas herramientas y equipamiento para las huertas. Sin embargo, el proceso de solicitud y cobro de dicho subsidio estuvo atravesado por diversas dificultades (tanto internas como para con el organismo público) que concluyeron en la obtención de herramientas distintas a las que habían solicitado en un primer momento, y que luego no fue posible aprovechar.

\section{Aspectos condicionantes de las posibilidades de continuidad de la experiencia}

Dado el funcionamiento que sostenía La Raíz, se pueden reconocer dificultades vinculadas a necesidades estructurales y aquellas propias de las relaciones, los vínculos personales y los acuerdos organizacionales.

Una de las principales limitantes que sus integrantes manifestaron fue la falta de un transporte adecuado y con capacidad suficiente para el traslado de la mercadería a las distintas localidades por las que itineraba la feria. El traslado se llevaba a cabo en autos personales, lo cual generaba serias 
dificultades cuando no se encontraban en adecuadas condiciones de uso. Si bien luego se avanzó en la compra de un "carro" para el traslado de la producción, las discusiones internas en torno a los acuerdos de su uso comunitario limitaron las posibilidades reales de mejora.

Asimismo, la falta de herramientas acordes y con capacidad de mantener el incremento en los niveles de producción que se estaba generando hacía que el trabajo en las huertas se realizara de la misma manera en que se producían menores cantidades. Esto no solo generó expectativas, sino también, por ejemplo, la inclusión de más integrantes de las familias de los productores, quienes finalmente terminaron por abandonar el grupo.

Este tipo de situaciones permite considerar las dificultades que presentan este tipo de experiencias para su sostenibilidad. Algunos de los entrevistados comentan al respecto que varios integrantes fueron desvinculándose de La Raíz ante otras posibilidades laborales que les brindaban mayores recursos económicos. Por otro lado, destacan que la intermitencia y la participación menos activa son propias de estas prácticas, en relación a que algunas personas simplemente enviaban su mercadería a través del referente y principal organizador de la feria sin involucrarse en las reuniones.

Estas diferentes visiones acerca de cómo se entendía el funcionamiento generó disconformidades, ya que La Raíz estaba constituida por muchas más personas de las que sostenían las diferentes actividades (reuniones, ventas, etc.), de modo que debían además compartir los espacios de venta, lo que reducía las posibilidades de comercializar mayores cantidades. Consecuentemente, algunos acuerdos que inicialmente se establecían en las reuniones, y fundamentalmente relacionados con los precios de venta, comenzaron a realizarse de modo individual con el referente y crearon circuitos paralelos de comunicación e intercambio. La existencia de un fuerte liderazgo en la organización, además, derivó en que en muchas de las actividades realizadas se impuso la impronta personal de quienes la presidían o coordinaban, frente a los acuerdos grupales alcanzados.

En síntesis, si bien son múltiples y variadas las dificultades que encontraron los participantes de La Raíz, se pueden destacar los desacuerdos internos y las falencias organizativas como elementos principales que condicionaron la posibilidad de continuidad de la experiencia.

\section{Transitando nuevos caminos: la reconfiguración en Familias Campesinas}

A partir de la situación antes descripta, muchos de los integrantes de La Raíz comenzaron a alejarse y algunos a priorizar otras actividades, de manera que la organización atravesó un momento de gran debilidad y desarticulación. Es en ese contexto que, bajo el interés de mantener el canal de ventas y al reconocer las coincidencias existentes, continuaron trabajando de manera conjunta un grupo reducido de personas, quienes conformaron Familias Campesinas. Este grupo presentaba una clara impronta familiar, y se conformó por cinco mujeres y tres hombres.

Por eso, cuando nosotros integramos el nuevo grupo fue apostar otra vez, integrar grupos que yo conocía que me aseguraban que producían de una manera (...) Coincidíamos en que inicialmente ellos decían que trabajábamos de una misma manera, ¿y por qué no trabajar juntas si hablamos el mismo idioma? Entonces yo pensé en armar la nueva organización. (Entrevistada 1, comunicación personal, 1 de julio de 2016)

Familias Campesinas era también una organización dedicada a la comercialización, por lo que la coordinación de sus integrantes se refería, fundamentalmente, a las modalidades de venta. Inicialmente, los acuerdos giraron en torno a las cantidades a vender de cada producto, su calidad, el comportamiento con el cliente, la presentación, la limpieza y las cuestiones relativas a la composición conjunta de paquetes de productos.

Por otro lado, y de forma simultánea, a nivel del trabajo productivo, en las huertas, cobraba especial relevancia el espacio del hogar y familiar. Las cinco familias que participaban de la organización producían alimentos en huertas agroecológicas, en las que trabajaban de manera natural y cultivaban una gran variedad de hortalizas. Si bien algunas de ellas tenían pequeñas extensiones que gestionaban de forma individual, la organización de la producción se daba, principalmente, mediante grupos familiares que compartían un mismo lote. Eran dos los núcleos familiares: uno integrado por tres familias y el otro por dos. De esta manera, las huertas principales se sostenían 
con el aporte de varios integrantes cuya participación y nivel de compromiso variaba de acuerdo con los arreglos familiares. En este sentido, se destaca particularmente el liderazgo y protagonismo femenino, tanto en la producción como a nivel de participación organizacional.

Respecto al modo de producir, este se adelantaba sin ningún tipo de agregado químico. El cuidado de la tierra se basaba en abonos verdes y compost hechos con desechos húmedos, aunque se mencionan los altos costos que los productos químicos tenían para el tipo y la escala de producción que ellos mantenían. Las herramientas utilizadas en general presentaban un bajo nivel de tecnificación, destacándose el pago por el uso de un tractor o algún móvil que traccione un disco para remover la tierra.

Se reconoce al mismo tiempo la existencia de un vínculo importante entre el cuidado de la tierra, la producción agrícola y las trayectorias de vida de las mujeres que formaban parte de la organización. Las más grandes de ambos grupos familiares reconocían su gratificación por el tipo de producción natural que realizaban, así como de la tarea que implicaba, y lo asociaban también con sus experiencias previas en la vida de campo. Incluso, se reconocía como elección de un modo de vida relacionado al cuidado de la salud, ante problemas que el tipo de producción convencional les había generado. Sin embargo, se observan ciertas contradicciones entre las ideas que se desprenden de sus relatos y sus prácticas cotidianas más allá de la huerta.

En términos de vínculos institucionales, desde sus comienzos estas familias contaron con el apoyo de un grupo de docentes, estudiantes y graduados de la Universidad Nacional del Litoral (UNL), quienes por medio de un proyecto de extensión de dicha universidad adelantaron diversas acciones tendientes al fortalecimiento de esta organización. Asimismo, algunos de los integrantes eran socios de una organización civil de alcance departamental (con reciente personería jurídica), a través de la cual gestionaron subsidios y créditos. Es por medio de distintos programas de fortalecimiento a este tipo de iniciativas que pudieron acceder a créditos que les permitieron comprar herramientas y maquinarias para su actividad, dada la imposibilidad de hacerlo a través del sistema financiero tradicional.

\section{Arreglos familiares de producción y reproducción: entre el trabajo para el mercado y el hogar}

En relación con la organización de las tareas, y de forma coincidente con la participación en Familias Campesinas, se observaba cierto liderazgo femenino en la asignación de tareas y coordinación de las huertas. La participación de integrantes de la familia en estas podía ser sistemática a través de tareas como el riego, la reproducción de semillas, el sembrado y trasplante, armado de plantines, etc., o bien podían tener una asignación y acuerdo específico por tareas puntuales, como, por ejemplo, el arado. En particular, esa tarea se asignaba a hombres $y$, de ser posible, se utilizaba algún tractor o vehículo para remover la tierra. Asimismo, se podía llegar a contratar a un tercero a quien se le pagaba por el combustible.

Esta diferenciación en grados de participación y tareas podría asociarse con las diferentes situaciones en que se encontraban las personas, la condición de género y los modos de subsistencia que tenían estas familias, ya que la pluriactividad es una característica común que les posibilitaba combinar diversas actividades con la producción huertera.

Lo antes mencionado se observa en aquellos casos en que la huerta no era la actividad principal de una persona, lo cual ocurre en general al poseer una relación laboral de dependencia o "changas" vinculadas a la producción agrícola en otras quintas, incluso de otros familiares. Si bien los hombres eran quienes principalmente presentaban este tipo de situación, también se identificó el caso de una mujer que trabajaba en la quinta de un tercero. Incluso, la trayectoria de vida de muchas de las integrantes de Familias Campesinas está vinculada en distintos momentos a las actividades laborales en relación de dependencia en la producción de frutilla.

Sumado a esto, se identifica que la edad y la situación familiar es una variable de diferenciación del grado de participación en la huerta. Esta se vincula a la necesidad de los más jóvenes de compatibilizar las tareas productivas, bien sea porque mantenían estudios, o por la presencia de hijos pequeños que requerían cuidados, provistos por las jóvenes madres. 
En ambos sentidos, la organización familiar de la producción resulta una forma que les permitía combinar tareas y apoyar las diversas situaciones personales, al generar acuerdos internos familiares que eran variados y permitían la subsistencia.

Con la otra mi hija sembrábamos mucha sandía, melón, y las berenjenas (...) ella tuvo que empezar de nuevo con la otra pareja, hacer su casa. Entonces la única forma yo le dije es trabajar así, y bueno ahí empezamos, (eran) más cosas que llevaba el transporte. (Entrevistada 3, comunicación personal, 2 de julio de 2016)

\section{Refiere otra entrevistada:}

El año pasado habían dejado de estudiar para ayudarnos, Lorena y Martín mi yerno (...) Pero este año retomaron los estudios, porque se dieron cuenta que la venta en sí no redituaba mucho. Es decir, elegimos una profesión para ayudar en la economía de los hogares, entonces nosotros dijimos sí... vamos a producir un poco más para poder vender y paliar los gastos. (Entrevistada 1, comunicación personal, 1 de julio de 2016)

Es notoria también la diversificación de actividades productivas de tres mujeres que pertenecían a un mismo grupo familiar (que contaba con mayores vulnerabilidades socio-económicas), quienes, además de trabajar en la huerta, bien fuera como actividad principal o bien secundaria, producían alimentos caseros tales como panificados, dulces, escabeche, conservas y otros derivados de las producciones del hogar, los cuales se vendían junto con las hortalizas, pero también por fuera de la organización. Estas actividades, que además de sumar ingresos sumaban horas de trabajo, se vinculan con tareas feminizadas que se realizaban en el interior del hogar para la venta al mercado, e incluso se mantenían los fines de semana.

La descripción hasta el momento señalada permite visualizar de qué manera el trabajo productivo, bien sea realizado en el ámbito doméstico-familiar o no, se combina con otras múltiples actividades. La dedicación horaria a esos trabajos se condice con las diversas modalidades de inserción y las limitaciones antes mencionadas. Sin embargo, al tiempo dedicado por algunas de las mujeres a los trabajos productivos, deben agregarse aquellas labores dedicadas al mantenimiento de la casa y el cuidado.
Yo me levanto a las 6. A las 6, tomo mate con mi esposo. Y si tengo pedido pan, hago pan porque hago pasta frola, lemon pie, tengo pedido (...) Bueno hago todo lo que tengo que hacer mientras es oscuro. Cuando ya aclaró todo le doy de comer a los perros, a los chanchos, y bueno de ahí yo ya me voy a la quinta (...) A la tarde, como siembro a media con Marina, a la tarde trabajamos con ella en el otro pedazo, hasta las 4, las 5. (Entrevistada 3, comunicación personal, 2 de julio de 2016)

El trabajo doméstico y de cuidado realizado en estos hogares, en su mayoría por mujeres, generaba una rutina intensa, en la que lo productivo y lo reproductivo se fundía en una trama de difícil disociación. Los aspectos reproductivos toman cuerpo, como ya se mencionó, no solo en el trabajo realizado en el hogar, sino también en las posibilidades de acceso de estas mujeres al trabajo mercantil, desde la propia generación de trabajos considerados "femeninos".

\section{Desafíos para la sostenibilidad de Familias Campesinas}

Una de las principales dificultades que se observaron en este grupo es la complejidad de la coordinación, debido a la forma de comercialización y de organización para el tipo de productos que vendían. Las huertas de cada familia, en general, presentaban una diversidad de hortalizas, por lo que generar acuerdos sobre el tipo y la cantidad de productos que ofrecía cada integrante no era un tema menor. Sin embargo, no se observaba interés ni viabilidad en coordinar el tipo de producción de cada familia, ya que Familias Campesinas no era el único canal comercial que mantenían y, por tanto, se valoraba la diversidad de productos.

Esta situación se relaciona con otra de las principales dificultades observadas: el modo en que se repartían los ingresos. La tensión se producía al momento de considerar la retribución por el trabajo de cada integrante. Si bien en principio se dividían los ingresos de manera equitativa entre ellos, hay quienes planteaban la necesidad de que se les retribuyera según lo que cada uno aportaba. Estas posturas divergentes son resultado de la misma organización por grupos familiares que mantenían la mayoría de las huertas, ya que el control o individualización del trabajo de cada uno resultaba 
confusa y generaba tensiones. Además de esto, la importancia del emprendimiento para el sustento familiar no era la misma para cada uno de los miembros del grupo (se trataba en algunos casos del principal ingreso, mientras que en otros de un complemento), lo que derivó en diversas oportunidades en desacuerdos acerca de la fijación de los precios, ya que estos brindaban la posibilidad de obtener ventajas diferenciales.

En relación con la rentabilidad y los ingresos que generaba la actividad, los productores se mostraban conformes con el nivel de ventas, pero reconocían que el transporte de los productos a zonas aledañas implicaba la necesidad de analizar más en profundidad los costos, a fin de conocer su viabilidad en el futuro.

Por último, los productores reconocían el riesgo propio del tipo de actividad productiva que desarrollaban, ya que se encuentra sujeta al clima. Los fuertes temporales que ocurren en distintas zonas de la provincia de Santa Fe y que, en particular, afectan al sector agrícola, deterioran no solo las huertas, sino también los hogares de estas familias, y llegan incluso a limitar en algunas ocasiones la posibilidad de reproducir las semillas (forma habitual de conseguirlas), lo cual representa una importante dificultad para las siguientes siembras.

\section{Reflexiones finales}

Al recuperar el concepto de sostenibilidad plural, podemos reflexionar acerca de las distintas dimensiones que atraviesan esta experiencia y posibilitan o no su permanencia en el tiempo.

En este tipo de organizaciones asociativas y autogestivas la sostenibilidad no está dada simplemente por su capacidad de competir en el mercado - y reducir así el análisis a una ecuación costos-beneficios-, sino que implica observar múltiples dimensiones. Resulta indispensable en ese sentido considerar los lazos y vínculos de confianza que se generan al interior de estas y permitan sostener arreglos asociativos y la cohesión grupal, así como también el aporte y el vínculo que el ámbito privado del hogar tiene con estas experiencias, en tanto ámbito en que confluyen los trabajos de producción y de reproducción que posibilita sostener los niveles de vida de los integrantes.

Bajo esta perspectiva, podemos entonces reconocer, a partir de la trayectoria analizada, cómo algunos de los elementos favorables a la permanencia de las organizaciones se vinculan a la dimensión política, destacándose los vínculos con instituciones y organizaciones sociales y estatales. Si bien estas redes generan contención, apoyo e intercambio, por sí solas no permiten un salto cualitativo en las prácticas de la Esys. Asimismo, se destacan las coincidencias relativas a las prácticas de producción sustentables en términos ambientales, en tanto persiguen el cuidado de la tierra y la alimentación saludable, pero también permiten forjar una identidad compartida por sus integrantes.

Por el contrario, aquellos aspectos que de algún modo se pudieron identificar como limitantes a la sostenibilidad están relacionados con las dimensiones económica, ambiental y asociativa de la sostenibilidad. En ese sentido, si bien se identifican necesidades estructurales de la actividad mercantil desarrollada, se destacan fundamentalmente dos situaciones: 1) la dificultad de mantener los acuerdos organizacionales y 2) la falta de ingresos suficientes o rendimientos diferenciales de sus integrantes, lo cual se combina con el surgimiento de otras oportunidades de trabajo. En relación con este último punto, eran los hombres quienes presentaban mayores posibilidades de compatibilizar su participación en las organizaciones de la Esys con trabajos en relación de dependencia, mientras que las mujeres generaban sus formas de autoempleo, caracterizadas por tareas feminizadas. Asimismo, la forma de organización y los modos de vida que mantenían estas familias hacían que los límites entre las esferas productiva y reproductiva se presentaran de modo difuso. Esto genera que la consideración del ámbito doméstico como espacio de trabajo en la Esys sea particularmente relevante, y la posibilidad de permanencia de esta experiencia autogestiva dependa principalmente del trabajo de las mujeres. Por otro lado, también podríamos considerar que esa necesidad de mantener múltiples trabajos, sumada a la forma de producción familiar, genera que primen acuerdos de índole intrafamiliar antes que los organizacionales-asociativos.

En ese sentido, entonces, cabe destacar que las experiencias de la Esys analizadas parecieran estar vinculadas a las múltiples estrategias de reproducción que adoptan las familias y los acuerdos internos que estas conllevan. Existen, asimismo, dificultades para lograr una real cohesión al interior de la organización, derivadas en algunos casos de la falta de confianza entre los participantes, así como de la dificultad para 
asumir compromisos y responsabilidades más allá de la producción propia. En general, como ya fue mencionado, priman acuerdos autogestivos individuales o familiares por sobre los asociativos, lo cual plantea importantes desafíos para la consolidación de prácticas que abonen al desarrollo de un sector de la Esys con reales posibilidades de resolver las necesidades de reproducción de quienes la integran.

\section{Referencias}

Abramovich, A. L. (2008). Emprendimientos productivos de la economía social en Argentina: funcionamiento y potencialidades. En A. Cimadamore (Comp.), La economía política de la pobreza. Buenos Aires: Clacso.

Abramovich, A. L. y Vázquez, G. (2004). La difícil construcción de una economía social. Los emprendimientos productivos de la economía popular. Conferencia llevada a cabo en el in Congreso Nacional de Políticas Sociales, Mendoza, Argentina.

Benería, L. (1981). Reproducción, producción y división sexual del trabajo. Mientras tanto, 6, 47-84

Carrasco, C. (2016). Sostenibilidad de la vida y ceguera patriarcal. Una reflexión necesaria. Atlánticas. Revista Internacional de Estudios Feministas, 1(1), 34-57. doi: https://doi.org/10.17979/arief.2016.1.1.1435.

Carrasco, C., Borderías C. y Torns, T. (2011). Introducción. El trabajo de cuidados: antecedentes históricos y debates actuales. En C. Carrasco, C. Borderías, y T. Torns (Eds.), El trabajo de cuidados. Historia, teoría y políticas (pp. 13-95). Madrid: Catarata.

Casalis, A. (2007). La economía social como subsistema y sus aportes al desarrollo local, en el marco del desarrollo. Buenos Aires: Mimeo.

Coraggio, J. L. (1998a). Introducción. En J. L. Coraggio (Ed.), Economía urbana. La perspectiva popular (pp. 7-12). Quito: Ediciones Abya-Yala.

Coraggio, J. L. (1998b). Posibilidad y sentido de una economía popular urbana. En J. L. Coraggio (Ed.), Economía urbana. La perspectiva popular (pp. 57-87). Quito: Ediciones Abya-Yala.

Coraggio, J. L. (2008). La sostenibilidad de los emprendimientos de la economía social y solidaria. Otra Economía, 2(3), 41-57.

De Oliveira, O. y Ariza, M. (1997). División sexual del trabajo y exclusión social. Revista Latinoamericana de Estudios del Trabajo, 3(5), 183-202.

Dos Santos, V. G., Matos-Filho, J., Medeiros de Araujo, M., Chaves Meireles, D., y Da Silva, J. A. (2013, ju- nio-diciembre). Do singular ao plural: indicadores de sustentabilidades na economía solidária. Opará -Etnicidades, Movimentos Sociais e Educação, 2.

França-Filho, G. y Laville, J. L. (2004). Economia solidária: uma abordagem internacional. Rio Grande do Sul: Editorial de la UFGs.

Gaiger, L. I. (2007). A outra racionalidade da economia solidária; conclusões do primeiro Mapeamento Nacional no Brasil. Revista Crítica de Ciências Sociais, 79, 57-77. doi: https://doi.org/10.4000/rccs.725.

Ghibaudi, J. (2003). Economía solidaria: su matriz conceptual y su objeto de estudio. Ponencia presentada en las Ix Jornadas de Epistemología de las Ciencias Económicas, Facultad de Ciencias Económicas, Universidad de Buenos Aires.

Hintze, S. (2009). Las políticas públicas en la sostenibilidad de los agentes y organizaciones de la economía social y solidaria: reflexiones a partir de la experiencia de Venezuela y Brasil. Conferencia llevada a cabo en el Seminario Internacional La construcción de conocimiento y prácticas sobre la economía social y solidaria en América Latina y Canadá, Buenos Aires, Argentina.

Hoop, M. (2012). La sostenibilidad de los emprendimientos asociativos y autogestionados. ORG \& DEMO, 2(2), 39-58.

Kraychete, G. (2012). Economia popular solidária: indicadores para qual sustentabilidade? En G. Kraychete y P. Carvalho (Org.), Economia popular solidária: indicadores para a sustentabilidade (pp. 15-25). Porto Alegre: Tomo Editorial.

Lisboa, A. M. (2005). Economia solidária e autogestão: imprecisão e limites. RAE. Revista de Administração de Empresas, 45(3), 109-115. doi: https://doi. org/10.1590/S0034-75902005000300006.

Matthaei, J. (2010). Más allá del hombre económico: crisis económica, economía feminista, y la economía solidaria. Cayapa. Revista Venezolana de Economía Social, 10(19), 65-80.

Picchio, A. (2001). Un enfoque macroeconómico ampliado de las condiciones de vida. Conferencia inaugural de las Jornadas Tiempos, Trabajos y Género. Facultad de Cs. Económicas, Universidad de Barcelona.

Polanyi, K. (2011). La gran transformación: los orígenes políticos y económicos de nuestro tiempo. Buenos Aires: Fondo de Cultura Económica.

Quiroga, N. (2010). ¿De qué crisis estamos hablando? Cuestionamientos y propuestas a la política de activos desde la economía feminista y la economía social. En J. L. Coraggio, y V. Costanzo (Eds.), Mentiras $y$ verdades del "capital de los pobres". Perspectivas desde la economía social y solidaria (pp. 277-326). Buenos Aires: Ediciones Imago Mundi-Ungs. 
Ray, C. (2000). Endogenous sócio-economic development and trustful relationship: partnerships, social capital and individual agency. Working Paper 45. Centre for Rural Economy, University of Newcastle upon Tyne.

Rodríguez-Enríquez, C. (2010). Análisis económico para la equidad: los aportes de la economía feminista. $P u$ blicación Saberes, 2, 3-22.

Salvia A. (2007). Consideraciones sobre la transición a la modernidad, la exclusión social y la marginalidad económica. Un campo abierto a la investigación social y al debate político. En A. Salvia, y E. Chávez Molina (Comps.), Sombras de una marginalidad fragmentada. Aproximaciones a la metamorfosis de los sectores populares de la Argentina. Buenos Aires: Niño y Dávila.

Sen, A. (2000). Desarrollo como libertad. Madrid: Editorial Planeta.

Singer, P. (2007). Economía solidaria: un modo de producción y distribución. En J. L. Coraggio (Org.), La economía social desde la periferia. Contribuciones latinoamericanas. Buenos Aires: UNGs- Altamira.

Vázquez, G. (2010). El debate sobre la sostenibilidad de los emprendimientos asociativos de los trabajadores autogestionados. Revista de ciencias sociales, segunda época, 18, 79-120. 